\title{
Burst behavior at a capillary tip: effect of low and high surface tension Damena D. Agonafer ${ }^{\text {a,* }}$, Ken Lopez a , James W. Palko ${ }^{\text {a }}$, Yoonjin Won ${ }^{\text {a }}$, Juan G. Santiago ${ }^{\text {a }}$, Kenneth E. Goodson ${ }^{\text {a }}$ \\ ${ }^{a}$ Department of Mechanical Engineering, Stanford University 440 Escondido Mall, Bldg. 530, Room 224, Stanford, CA, 94305-3030, USA
}

Corresponding Author Tel.: +1 650-285-7396; fax: +1 650-285-7396

E-mail address: agonafer@stanford.edu (D.D. Agonafer)

\begin{abstract}
Liquid retention in micron and millimeter scale devices is important for maintaining stable interfaces in various processes including bimolecular separation, phase change heat transfer, and water desalination. There have been several studies of re-entrant geometries, and very few studies on retaining low surface tension liquids such as fluorocarbon-based dielectric liquids. Here, we study retention of a liquid with very low contact angles using borosilicate glass capillary tips. We analyzed capillary tips with outer diameters ranging from 250-840 $\mu \mathrm{m}$ and measured Laplace pressures up to $2.9 \mathrm{kPa}$. Experimental results agree well with a numerical model that predicts burst pressure (the maximum Laplace pressure for liquid retention), which is a function of the outer diameter $(D)$ and capillary exit edge radius of curvature $(r)$.
\end{abstract}

KEYWORDS: Canthotaxis Effect; Liquid Pinning; Superomniphobic Surfaces; Phase Separation; Low Surface Tension Liquids. 


\section{Nomenclature}

$\mathrm{Ca}$ capillary number

D capillary outer diameter, $\mathrm{m}$

$d \quad$ capillary inner diameter, $\mathrm{m}$

$N \quad$ number of tracking points

$p \quad$ pressure, $\mathrm{Pa}$

$R \quad$ radius of curvature of meniscus, $\mathrm{m}$

$r \quad$ radius of curvature of capillary edge, $\mathrm{m}$

$U \quad$ liquid front velocity $(\mathrm{m} / \mathrm{s})$

Greek Symbols

$\beta \quad$ meniscus center-point angle $\left({ }^{\circ}\right)$

$\gamma \quad$ surface tension $(\mathrm{N} / \mathrm{m})$

$\varphi \quad$ angular location of triple line $\left(^{\circ}\right)$

$\mu \quad$ dynamic viscosity $(\mathrm{kg} / \mathrm{m} \mathrm{s})$

$\theta \quad$ equilibrium contact angle $\left({ }^{\circ}\right)$

\section{Subscripts}

$N W \quad$ non-wetting

$W \quad$ wetting

\section{Introduction}

Passive stop valves (i.e. structures with no moving parts) have been used in microfluidics for restricting flow in microchannels via sudden expansions of a microchannel [1-4]. Rapid expansion of a microchannel produces a nozzle-like structure which can serve as a barrier for advancing liquid, requiring a decrease in radius of curvature and corresponding increase in pressure for a meniscus to enter into the expansion [5]. Strongly wetting liquids require larger expansion angles to exhibit adequate burst pressures [5-7]. Capillary stop valves have been shown to restrict the advance of aqueous liquids containing surfactants by varying the expansion angle along the capillary exit edge $[1,8,9]$. However, these studies demonstrated only pinning for liquids with finite equilibrium contact angles.

Highly wetting liquids, including dielectric liquids, have low surface energies and infinitesimal contact angles $\left(\sim 0^{\circ}\right)$ for all substrates of interest [10]. This makes it challenging to produce the convex menisci necessary to produce positive Laplace pressures. However, proper surface geometry can allow for liquid menisci to transition from concave to convex in shape, and so can be tailored to pin wetting liquids. For wetting liquids along a sharp-edged interface, any 
and outer diameter, $D$, that ranged from $250-840 \mu \mathrm{m}$. The objective of the experiments was to measure maximum liquid retention (burst) pressure and study the conditions before and after bursting through glass capillary tips. Pressure measurements were accompanied by simultaneous visualizations of the menisci using an optical microscope (Nikon Eclipse 80i) with a Plan Fluorite objective and numerical aperture (NA) of 0.15 . The bursting events were visualized in the region of the glass capillary tip and recorded using a CCD camera (Thorlabs DCC1545M), see Supplementary Material (Appendix B). The setup included a programmable syringe pump (Harvard PHD 2000) which pumped liquid through stainless steel tubing (IDEX) with inner diameter $62.5 \mu \mathrm{m}$, connected in series with a pressure transducer (Omega PX419) with 0-5 psig range, see Supplementary Material (Appendix B). The flow rates used in all experiments resulted in capillary numbers of $C a \leq 10^{-5}$, where

$C a=\frac{\mu U}{\gamma}$,

$\mu$ is the dynamic viscosity, $U$ is the liquid front velocity, and $\gamma$ is the liquid-vapor surface tension. The liquids used for these studies were deionized (DI) water, Fluorinert (FC-40) (Sigma-Aldrich, St. Louis, MO, USA, CAS Number: 51142-49-5), and Isopropyl alcohol (IPA) (Sigma-Aldrich, St. Louis, MO, USA, CAS Number: 67-63-0), See Supplementary Material (Appendix C) for thermophysical properties for each liquid.

\section{Theory and Model Results}

To study bursting events through capillary tips, we developed a theoretical model to demonstrate the mechanism for pinning liquids along the rounded outer edge of a capillary. Figure 1 is a schematic of the capillary model in cross section. The primary geometric parameters for the model are capillary outer diameter $(D)$ and edge radius of curvature $(r)$. The 
location of the contact line of the solid-liquid-vapor interface is given by the angular coordinate $\varphi$ defined with respect to the exit edge curvature, $r$ (c.f. Fig. 1). The three-dimensional capillary geometry is defined by the revolution of the cross-section about its centerline. The model only considers capillary dominated flow (i.e. $C a \leq 10^{-5}$ ) and neglects any inertial, viscous, or gravitational forces. Therefore, the meniscus forms a spherical cap. The model and the following derivation were modified from a previous study on pinning liquids at the interfaces of microposts $[6,7]$.

The minimum radius of curvature of the meniscus $\left(R_{\min }\right)$ corresponds to the burst pressure, defined as the maximum Laplace pressure. The burst pressure occurs when the meniscus is in contact with the rounded outer capillary edge with curvature $r$ as illustrated in Fig. 1. To determine the value of burst pressure, the radius of curvature, $R$, of the meniscus forming a circular arc is calculated for various locations, $\varphi$, as:

$R(\varphi)=\frac{r \sin (\varphi)+\frac{D}{2}-r}{\sin (\beta)}$

where $\beta=\theta+\varphi$.

At each $\varphi$, the liquid forms the equilibrium contact angle $\theta$ with the solid surface, satisfying equilibrium conditions. For simplicity, the equilibrium contact angle is used instead of the advancing contact angle [18]. The Young-Laplace (Y.L.) equation defines the capillary pressure across a meniscus as inversely proportional to $R$,

$\Delta p=p_{W}-p_{N W}=\frac{2 \gamma}{R}$

The burst pressure is defined as the pressure value at the angular location, $\varphi$, associated with the minimum radius of curvature of the meniscus, $R_{\min }$. The location of $R_{\min }$ in terms of $\varphi$ is dependent on the surface tension of the liquid. The re-entrant surface geometry of the capillary 
tip forms a pressure barrier for both high and low surface tension liquids. This geometry allows the meniscus to transition to a convex shape, resulting in a positive Laplace pressure $\left(p_{W}>p_{N W}\right)$. The angular location of the minimum radius of curvature for liquids with finite contact angles should always occur at $\varphi<90^{\circ}$. However, liquids with infinitesimal contact angles (e.g. alkane and fluorocarbon-based dielectric liquids) will result in an angular location of $R_{\min }$ at $\varphi=90$.

Figure 2 shows model predictions, for FC-40 and water, of meniscus shape (Fig. 2a) and pressure versus meniscus location (Fig. 2b) as the meniscus advances along an edge with radius of curvature, $r=1 \mu \mathrm{m}(r<<)$, of a capillary tip with outer diameter $(D)$ of $550 \mu \mathrm{m}$ and inner diameter $(d)$ of $400 \mu \mathrm{m}$. The model is evaluated at ten values of $\varphi$. The solid black lines in Fig. 2a indicate the meniscus with $R_{\min }$ corresponding to the bursting pressure for each liquid. The dotted gray lines indicate meniscus shape at lower pressures. Figure $2 \mathrm{~b}$ shows pressure versus angular location, with the location corresponding to burst indicated by the open diamond symbols. Figure 2 shows that the minimum radius of curvature, $R_{\min }$, for FC-40 occurs at $\varphi=90^{\circ}$ corresponding to a bursting pressure of $0.12 \mathrm{kPa}$. Since the contact angle for FC-40 on glass is $\sim 0^{\circ}$, the bursting event will occur at $\varphi=90^{\circ}$ for capillaries irrespective of the capillary edge curvature, $r$. Similar results are seen for IPA, which has a contact angle with glass of $\sim 0^{\circ}$. For water, with a contact angle on borosilicate glass of $\sim 60^{\circ}$, the burst pressure occurs at $\varphi=27^{\circ}$, prior to the meniscus advancing to the outer capillary surface (i.e. $\varphi=90$ ). Figure $2 \mathrm{~b}$ illustrates that the burst pressure for water is $0.53 \mathrm{kPa}$. Although the radius of curvature of water, $R$, is at a minimum, there's still a finite distance for the meniscus to expand along the outer capillary edge. The pressures due to the continued growth past the bursting pressure is lower as the radius of curvature, $R$, increases and the droplet expands in size as shown in Fig. 2a for water. 
These results suggest that for FC-40 and IPA, which have a contact angle of $\sim 0^{\circ}$, the edge radius of curvature, $r$, does not affect the burst pressure and location of the minimum radius of curvature, $R_{\min }$. We attribute this to the wetting behavior of low surface tension liquids with infinitesimal contact angles. Therefore, the outer diameter, $D$, is the only critical dimension for pinning low surface energy liquids along a capillary edge. In contrast, the burst pressure for water can show dependence on $r$ if $r$ is significant compared to $D$.

\section{Results and discussion}

\subsection{Burst pressure results for glass capillary tips}

Figure 3 illustrates image sequences from experimental visualizations of flow through capillary tips with $D=550 \mu \mathrm{m}$ for DI water, IPA, and FC-40. These experiments show expected signatures of burst for all liquids. As the system pressure is increased, the meniscus along the capillary edge expands followed by either a discernible drop in pressure or a prolonged plateau. All pressure measurements are referenced to the ambient pressure prior to activation of the flow. Liquid flows through the capillary tube with minimal viscous effects $\left(\mathrm{Ca} \leq 10^{-5}\right)$, and consequently, the pressure in the liquid varies only due to hydrostatic effects. During this period, $p_{W}$ in the liquid is lower than $p_{N W}$ (air) by an amount determined by the contact angle of the liquid and inner capillary diameter and given by Eq. 3 and illustrated in images a, f, k of Fig. 3 for FC-40, IPA, and water respectively. When the meniscus flows to the top capillary surface, it pins at the inner capillary edge with diameter, $d$, as shown in images $\mathrm{b}$ and $\mathrm{g}$ for FC-40 and IPA, respectively, in Fig. 3. At this point, the pressure in the liquid begins to rise as the meniscus becomes less concave. Eventually the meniscus becomes planar, consistent with equal pressure 
between the liquid and gas phases as shown in images $\mathrm{c}, \mathrm{h}$, and 1 , for FC-40, IPA, and DI water, respectively, in Fig. 3.

As flow continues, the meniscus can form a convex shape, and the pressure of the liquid $\left(p_{W}\right)$ rises above that of the gas $\left(p_{N W}\right)$. For FC-40 and IPA, the menisci do not become convex at the inner diameter of the capillary, instead spreading along the top surface of the capillary to the outer edge, due to their contact angles of $\sim 0^{\circ}$. As predicted by the model, the minimum radii of curvature for FC-40 and IPA occur at $\varphi=90^{\circ}$, which coincides with an angle of $180^{\circ}$ with the outer capillary edge. The menisci at maximum pressure for FC-40 and IPA are shown in Fig. 3, images $d$ and i, respectively. Any further volume of FC-40 and IPA delivered to the tip will lead to a burst event and subsequent dripping/flow of liquid down the outer surface of the capillary as shown in Fig. 3, images e, j. For water, the meniscus initially is pinned at the inner edge of the capillary as seen in Fig. 3, image m, due to its finite contact angle $\left(\sim 60^{\circ}\right)$. Further flow results in an increase in radius of curvature as the meniscus forms an advancing contact angle with respect to the top surface of the capillary. At this point, the meniscus flows to the outer edge of the capillary and again is pinned. Continued flow initially decreases the meniscus radius of curvature until a minimum is reached corresponding to the burst pressure as shown by the model (Fig. 2). Unlike FC-40 and IPA, further flow of water does not immediately advance down the outer edge of the capillary. Instead, the meniscus continues to expand, increasing its radius of curvature and decreasing the retained pressure (Fig. 3b), until it forms an angle with the outer capillary edge equal to the advancing contact angle (Fig 2). We attribute the sudden drop in pressure to $p_{W}$ overcoming the pressure barrier associated with the change in total interfacial energy $[4,9]$. 
We ascribe the contrasting bursting behavior between FC-40, IPA, and water to the differences in surface tension and vapor pressure for each liquid. Water will not 'de-pin' and spread down the outer surface of the capillary until the meniscus forms an advancing contact angle $\left(\sim 60^{\circ}\right)$ with the outer capillary edge. Therefore, the pressure/flow curves for water show a distinct maximum, see Supplementary Material (Appendix D). However, for lower surface tension liquids (e.g. FC-40, IPA), liquid 'runs' along the outer surface of the capillary when the meniscus is located at $\varphi=90^{\circ}$ (i.e. the meniscus forms a hemisphere), as can be noted in Fig. 3 for images $\mathrm{d}$ and $\mathrm{i}$. We hypothesize that the shape of the pressure/flow curve after burst is influenced by quasi-static and dynamic effects, effects influenced by thermophysical properties of the liquid and the flow rate, see Supplementary Material (Appendix D).

Figure 4 is a comparison between the numerical model predictions and experimental results for burst pressure. Plotted is Laplace pressure as a function of outer capillary diameter, $D$, for each liquid. The numerical model only predicts the pressure difference across the meniscus for cases where the meniscus wets the outer capillary diameter $(D)$. The model does not compute the additional capillary pressure due to the inner capillary diameter. Of course, the experimental pressure measurements include the canthotaxis effect due to both the inner and outer capillary edges [19].

To make a valid comparison, we here added to the predicted menisci pressure differences the capillary pressure associated with the inner capillary radius (as predicted by Eq. 3). We then plot this predicted total meniscus pressure in Fig. 4 and compare it to the experimental pressure measurements.

As expected, the Laplace pressure increases for decreasing capillary outer diameter, $D$. The capillary pressure is lowest for FC-40 due to the low surface tension of the liquid. Furthermore, 
FC-40 and IPA experiments demonstrate better agreement with the model than water. We hypothesize that imperfections and jagged edges along the rounded outer capillary edge may contribute to variability in burst pressures. The observed differences in burst pressure between liquids are well accounted for by surface tension.

\section{Conclusion}

We present a study of the retention of wetting liquids along the edge of the exit of a glass capillary. We developed a theoretical model that predicts the burst pressure along a rounded capillary edge. The model shows the outer capillary diameter, $D$, is the only critical dimension for retaining low surface tension liquids. The capillary experiments show excellent agreement with the numerical model that predicts the burst pressure along a rounded capillary edge. The experimental and theoretical model demonstrates that the bursting event for low surface tension liquids will occur when the meniscus is tangent to the outer capillary surface. Therefore for liquids with infinitesimal contact angles, a re-entrant surface geometry is required to achieve a convex meniscus and yield a positive Young-Laplace pressure $\left(p_{W}>p_{N W}\right)$.

This work demonstrates that retention of liquids with infinitesimal contact angles (e.g. fluorocarbon based dielectric liquids) can be achieved in nozzle-like structures with re-entrant surface curvature in contrast to previous work that designed superomniphobic surfaces for pinning low surface tension liquids on top of micropatterened structures $[17,20]$. The present work could be foundational for the development of novel repelling surfaces and membranes and microfabricated valving technologies.

Future interesting work may include studies of bursting pressure at higher temperatures (e.g., as applicable to heat transfer applications or other industrial processes), or studies of effects 
of roughness near the capillary edge. Further development of the capillary model will account for evaporation effects.

\section{Acknowledgments}

The authors would like to acknowledge financial support from DARPA (agreement \# HR0011-13-2-0011, titled: Phase Separation Diamond Microfluidics for HEMT Cooling) monitored by Avi Bar Cohen, Joe Maurer, and Kaiser Matin.

\section{References}

[1] A. Glière, C. Delattre, Modeling and fabrication of capillary stop valves for planar microfluidic systems, Sensors and Actuators A: Physical, 130 (2006) 601-608.

[2] H. Cho, H.-Y. Kim, J.Y. Kang, T.S. Kim, How the capillary burst microvalve works, Journal of colloid and interface science, 306 (2007) 379-385.

[3] M.G. Hospital, Capillary Flow, (2005) 192-197.

[4] P. Man, C. Mastrangelo, M. Burns, D. Burke, Microfabricated capillarity-driven stop valve and sample injector, in: Micro Electro Mechanical Systems, 1998. MEMS 98. Proceedings., The Eleventh Annual International Workshop on, IEEE, 1998, pp. 45-50.

[5] M. Zimmermann, P. Hunziker, E. Delamarche, Valves for autonomous capillary systems, Microfluid Nanofluid, 5 (2008) 395-402.

[6] J. Berthier, F. Loe-Mie, V.-M. Tran, S. Schoumacker, F. Mittler, G. Marchand, N. Sarrut, On the pinning of interfaces on micropillar edges, Journal of colloid and interface science, 338 (2009) 296-303.

[7] J. Berthier, V.-M. Tran, F. Mittler, N. Sarrut, The physics of a coflow micro-extractor: interface stability and optimal extraction length, Sensors and Actuators A: Physical, 149 (2009) 56-64.

[8] J.M. Chen, P.-C. Huang, M.-G. Lin, Analysis and experiment of capillary valves for microfluidics on a rotating disk, Microfluid Nanofluid, 4 (2008) 427-437.

[9] T.-S. Leu, P.-Y. Chang, Pressure barrier of capillary stop valves in micro sample separators, Sensors and Actuators A: Physical, 115 (2004) 508-515.

[10] K. Liu, X. Yao, L. Jiang, Recent developments in bio-inspired special wettability, Chemical Society Reviews, 39 (2010) 3240-3255.

[11] Y. Mori, T. Van de Ven, S. Mason, Resistance to spreading of liquids by sharp edged microsteps, Colloids and Surfaces, 4 (1982) 1-15.

[12] W. Choi, A. Tuteja, S. Chhatre, J.M. Mabry, R.E. Cohen, G.H. McKinley, Fabrics with tunable oleophobicity, Advanced Materials, 21 (2009) 2190-2195.

[13] Y. Kwon, N. Patankar, J. Choi, J. Lee, Design of Surface Hierarchy for Extreme Hydrophobicity, Langmuir, 25 (2009) 6129-6136.

[14] A. Susarrey-Arce, Á.G. Marín, S. Schlautmann, L. Lefferts, J. Gardeniers, A. van Houselt, One-step sculpting of silicon microstructures from pillars to needles for water and oil repelling surfaces, Journal of Micromechanics and Microengineering, 23 (2013) 025004.

[15] P.S. Forsberg, C. Priest, M. Brinkmann, R. Sedev, J. Ralston, Contact line pinning on microstructured surfaces for liquids in the Wenzel state, Langmuir, 26 (2009) 860-865. 
[16] A. Ahuja, J.A. Taylor, V. Lifton, A.A. Sidorenko, T.R. Salamon, E.J. Lobaton, P. Kolodner, T.N. Krupenkin, Nanonails: A Simple Geometrical Approach to Electrically Tunable Superlyophobic Surfaces, Langmuir, 24 (2007) 9-14.

[17] A. Tuteja, W. Choi, M. Ma, J.M. Mabry, S.A. Mazzella, G.C. Rutledge, G.H. McKinley, R.E. Cohen, Designing superoleophobic surfaces, Science, 318 (2007) 1618-1622.

[18] J. Oliver, C. Huh, S. Mason, Resistance to spreading of liquids by sharp edges, Journal of Colloid and Interface Science, 59 (1977) 568-581.

[19] T. Ondarçuhu, Total or Partial Pinning of a Droplet on a Surface with a Chemical Discontinuity, Journal de Physique II, 5 (1995) 227-241.

[20] T.L. Liu, C.-J.C. Kim, Turning a surface superrepellent even to completely wetting liquids, Science, 346 (2014) 1096-1100.

\section{Appendix A. Figures}

Fig. A1. Schematic of capillary model for a tip defined by outer diameter, $D$, and angle $\beta$ defined as the angle between the centerline and the tangent edge to the meniscus radius of curvature, $R$. The three-dimensional pore geometry is defined by revolving the cross-section about its centerline. The model only considers capillary flow (i.e. $C a \leq 10^{-5}$ ) and neglects gravitational, inertial, and viscous forces.

Fig. A2. Numerical results for: (a) Meniscus shape for a meniscus pinned along the outer edge of a capillary tip with inner diameter $d=400 \mu \mathrm{m}$ and outer diameter, $D=550 \mu \mathrm{m}$ for FC-40 and water. While pinned along the outer capillary edge, the pressure increases until bursting occurs, defined as the maximum achieved Laplace pressure. The dotted gray lines indicate the change in meniscus shape as a function of $\varphi$. The solid black lines indicate the minimum radius of curvature of the meniscus $\left(R_{\min }\right)$ that corresponds to the burst pressure. (b) Capillary pressure as a function of $\varphi$, defined as the angular location of the triple line along the edge radius of curvature, $r$. The open diamond symbols indicate the angular location $(\varphi)$ of the bursting event along the capillary edge. Since the contact angle for FC-40 on glass is $\sim 0^{\circ}$, the bursting event will occur when the meniscus is tangent to the outer capillary surface $\left(\varphi=90^{\circ}\right)$ for capillaries irrespective 
of the edge radius of curvature, $r$. For water, the bursting event occurs before the meniscus advances to the outer capillary edge $\left(\varphi=27^{\circ}\right)$. The water droplet expands beyond $R_{\min }$ until the meniscus forms an advancing contact angle with the outer capillary surface as shown in (a). The expansion of $R$ for water therefore subsequently leads to a decrease in Laplace pressure shown in (b).

Fig. A3. Image sequences of meniscus growth through a borosilicate glass capillary tip with inner diameter $d$ of $400 \mu \mathrm{m}$ and outer diameter $D$ of $550 \mu \mathrm{m}$ for FC-40 (images a-e), IPA (images f-j) and DI water (images k-o). The bursting pressures for FC-40 and IPA correspond to hemispherical menisci tangent to the outer surface of the capillary $(\mathrm{d}, \mathrm{i})$. Subsequent to this, the liquids continue and 'run' along the surface of the capillary as soon as the menisci become tangent with the outer surface. The meniscus for water, however, can expand beyond a hemisphere and pin along the outer edges of the capillary tip until the meniscus forms an advancing contact angle $\sim 60^{\circ}$.

Fig. A4. Comparison of analytical and experimental burst pressures for capillary tip outer diameters, $D$, ranging from $250-840 \mu \mathrm{m}$ for the menisci of DI water, IPA, and FC-40. FC-40 and IPA experiments have better agreement with the capillary model than water. We hypothesize that this is due to the variability of the edge radius of curvature, $r$, of the unpolished glass capillaries, which can lead to higher burst pressures. 


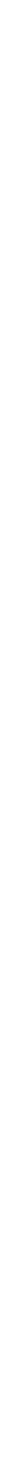


FC-40
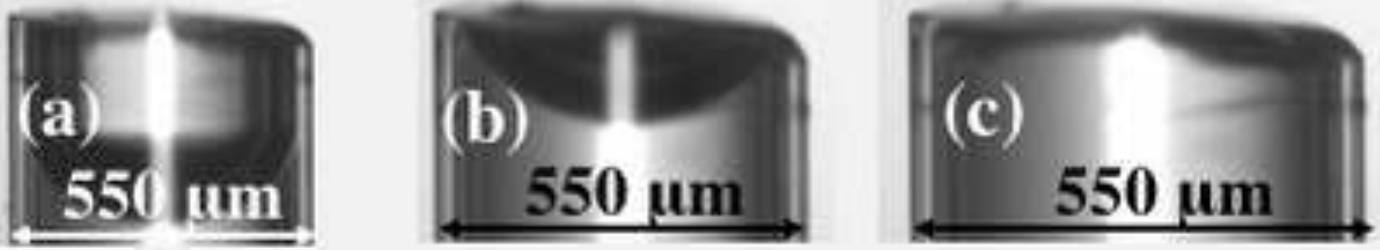

\section{Water}

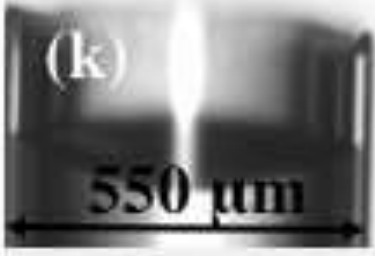

(d) $550 \mu \mathrm{m}$
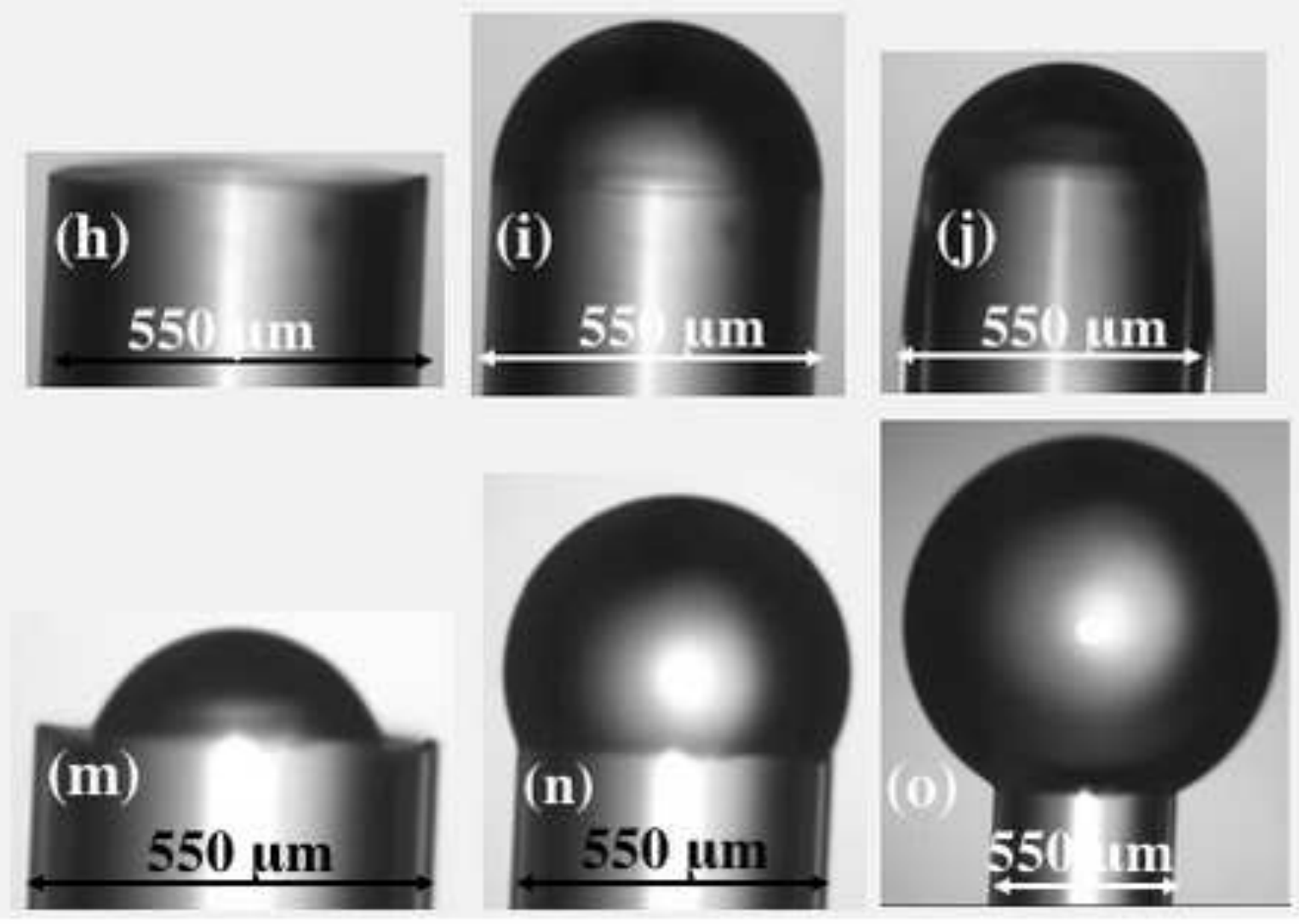

(e) $550 \mathrm{um}$

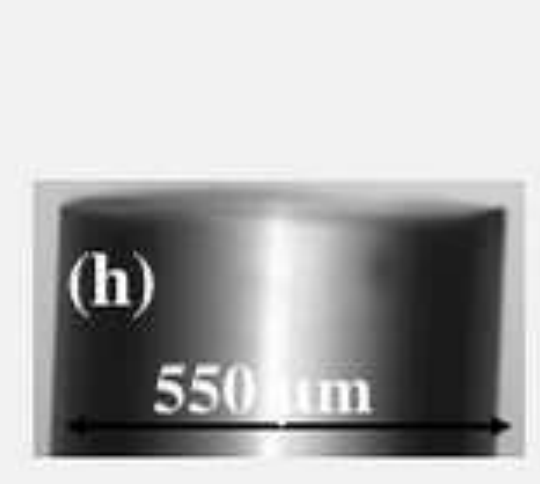

$550 \mu \mathrm{m}$
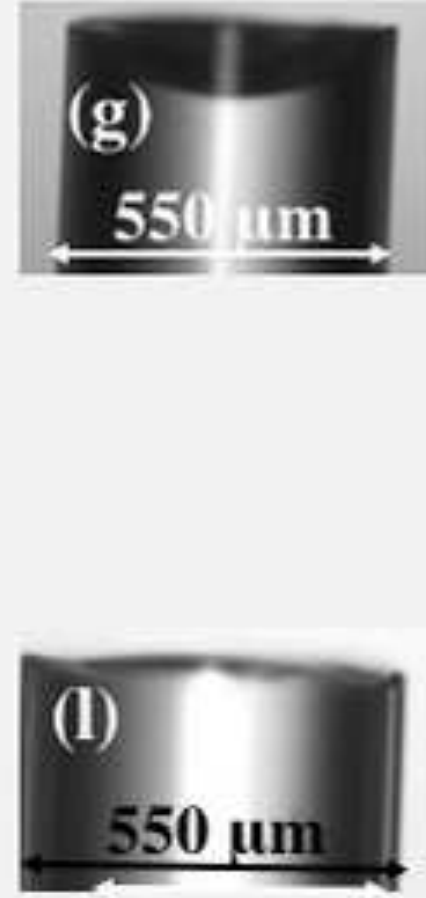
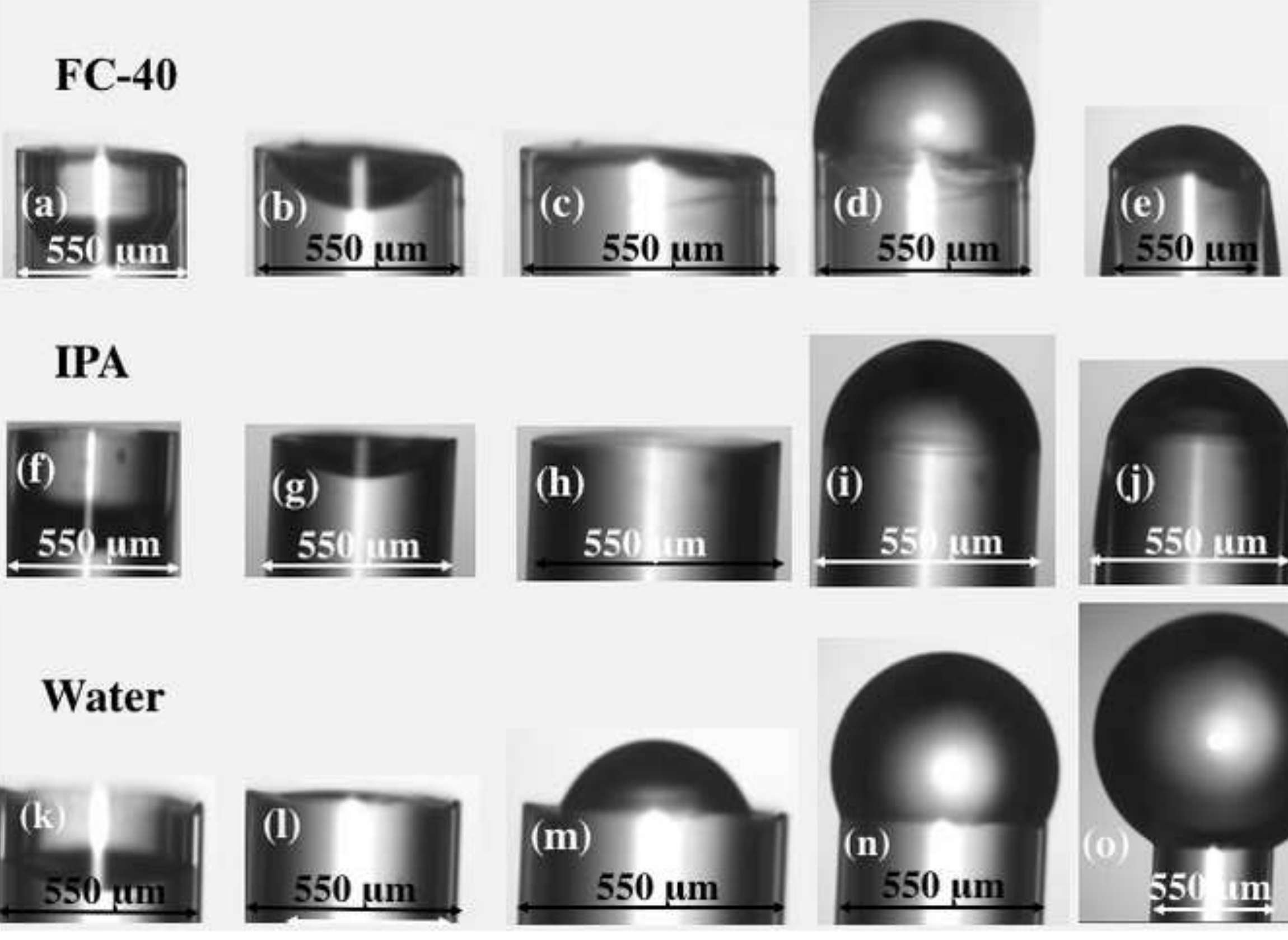


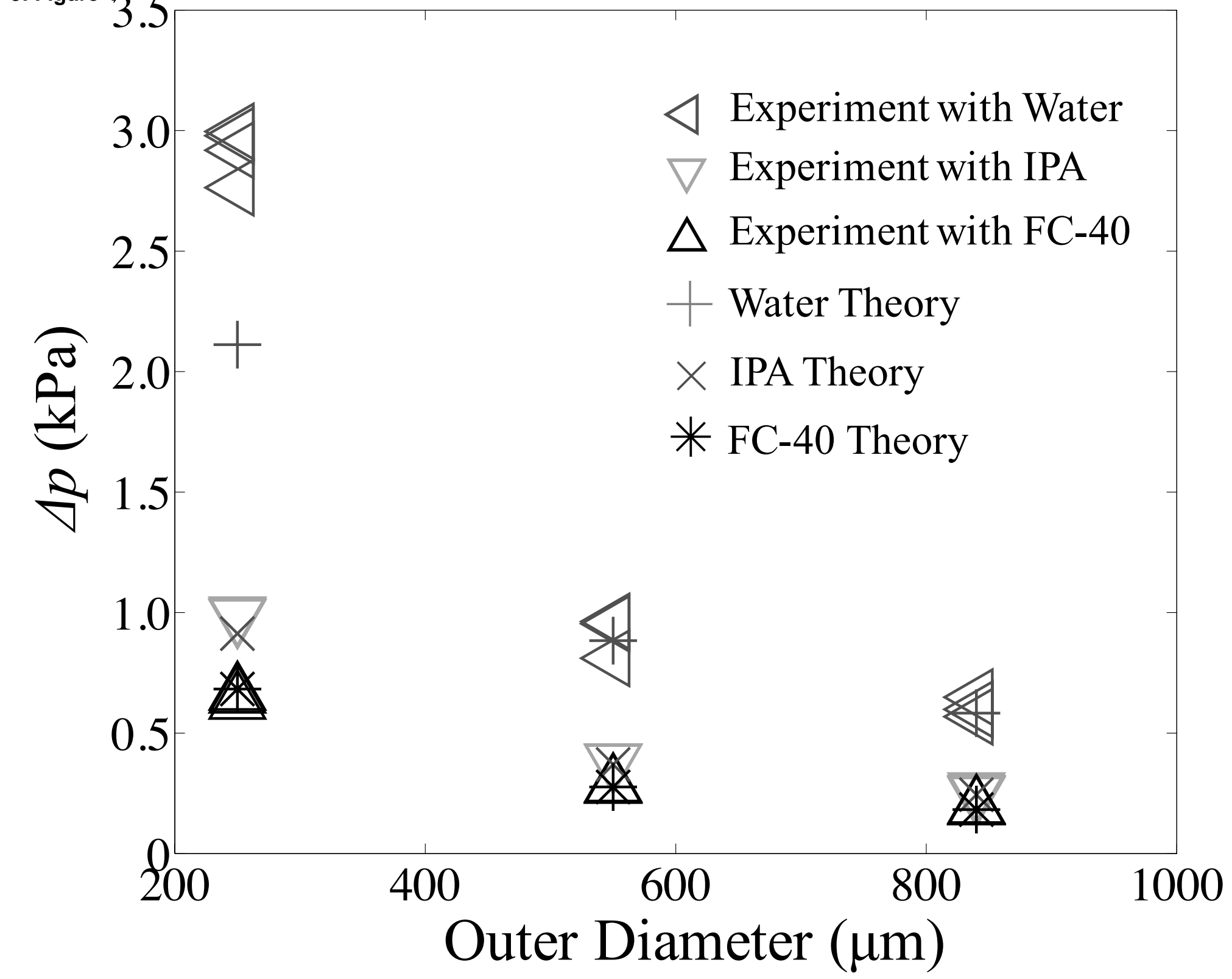



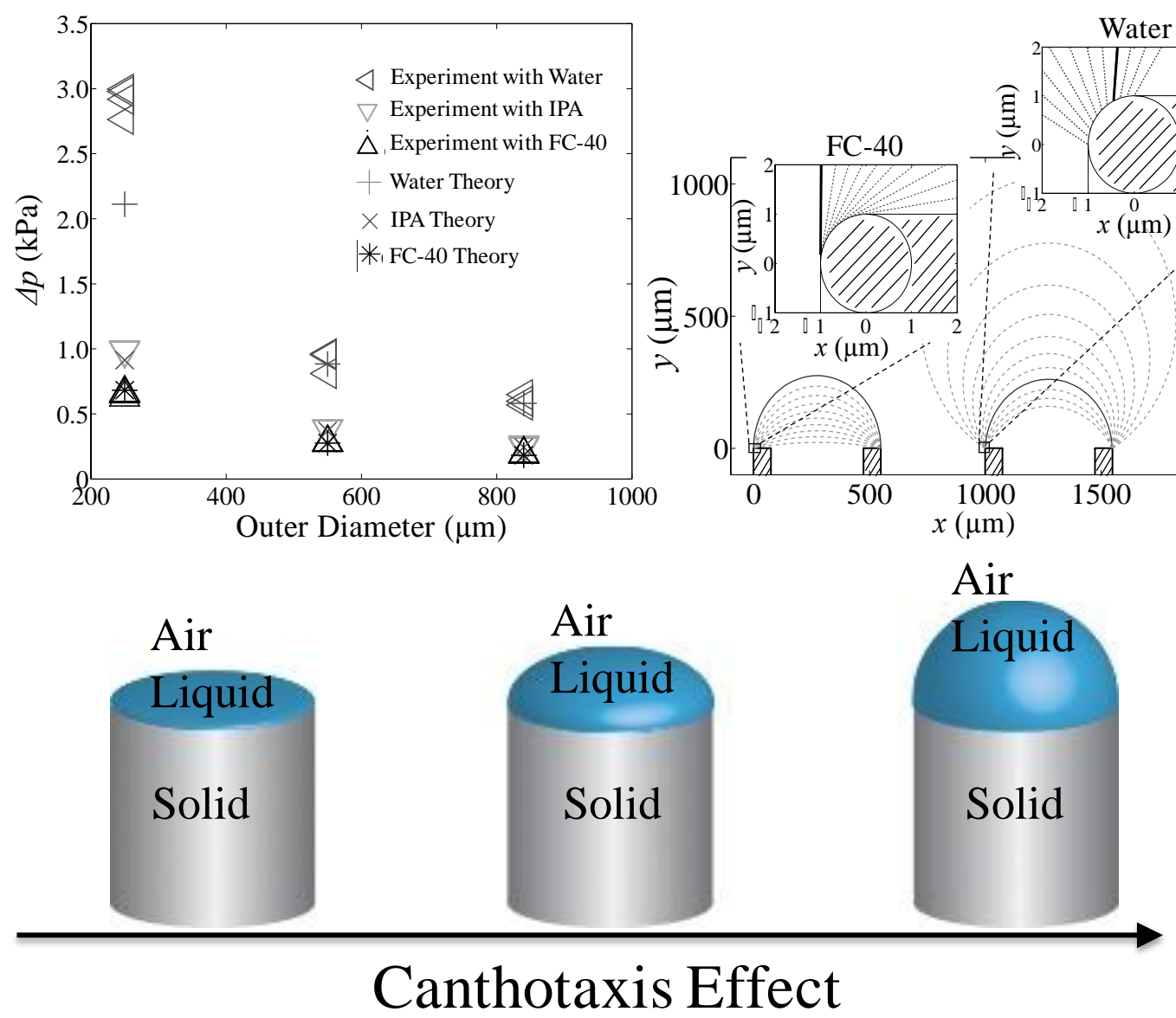

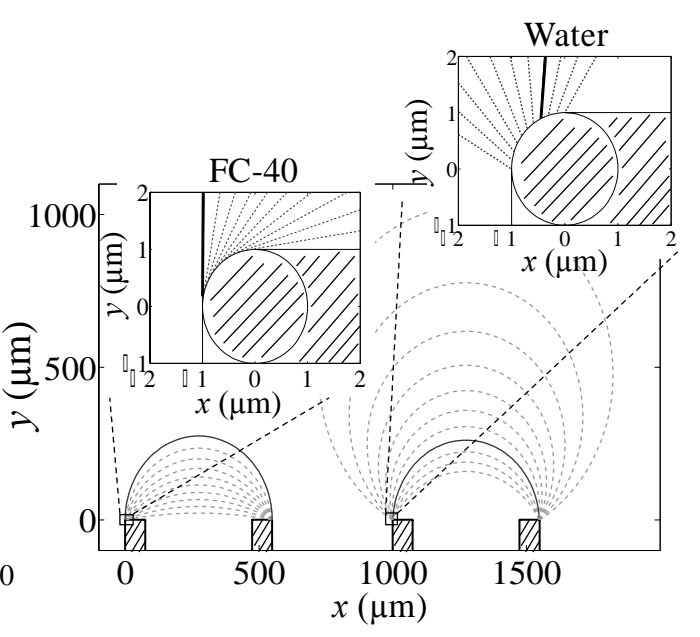

Air

\section{Canthotaxis Effect}

